\title{
A study on locating the sonic source of sinusoidal magneto-acoustic signals using a vector method
}

\author{
Shunqi Zhang, Xiaoqing Zhou, Ren Ma, Tao Yin and Zhipeng Liu* \\ Institute of Biomedical Engineering, Chinese Academy of Medical Sciences \& Peking Union Medical \\ College, Tianjin 300192, China
}

\begin{abstract}
Methods based on the magnetic-acoustic effect are of great significance in studying the electrical imaging properties of biological tissues and currents. The continuous wave method, which is commonly used, can only detect the current amplitude without the sound source position. Although the pulse mode adopted in magneto-acoustic imaging can locate the sonic source, the low measuring accuracy and low SNR has limited its application. In this study, a vector method was used to solve and analyze the magnetic-acoustic signal based on the continuous sine wave mode. This study includes theory modeling of the vector method, simulations to the line model, and experiments with wire samples to analyze magnetoacoustic (MA) signal characteristics. The results showed that the amplitude and phase of the MA signal contained the location information of the sonic source. The amplitude and phase obeyed the vector theory in the complex plane. This study sets a foundation for a new technique to locate sonic sources for biomedical imaging of tissue conductivity. It also aids in studying biological current detecting and reconstruction based on the magneto-acoustic effect.
\end{abstract}

Keywords: Magneto-acoustic effect, vector method, sonic source locating, amplitude, phase

\section{Introduction}

This study proposes a new method based on the magneto-acoustic effect for imaging electrical parameters of biological tissue [1] and sensing biological micro-currents [2].

In this magneto-acoustic method, tissue was placed in a magnetic field, and an electrode or coil produced a time-varied current. Then, the vibrations produced by Lorentz force [3-5] emitted magneto-acoustic (MA) signals, which could be detected and measured outside the sample. Thus, measuring the MA signal can reveal the conductivity [6] or the tissue's current [7].

In this method, electrical parameters of biological tissue samples were converted into a sonic signal. Measuring sonic waves is easier than measuring electromagnetic waves because sonic waves transmit at a much slower rate. Also, this method can avoid the dispersion effect caused by an electrical current. As a result, the magneto-acoustic method makes it possible to locate the sonic source with high

\footnotetext{
* Address for correspondence: Zhipeng Liu, Institute of Biomedical Engineering, Chinese Academy of Medical Sciences \& Peking Union Medical College, No. 236 Baidi Road, Nankai District, Tianjin 300192, China. Tel.: +8622-87891982; Fax: +8622-87892951; E-mail: lzpeng67@163.com.
} 
precision.

The current magneto-acoustic imaging method uses high voltage short pulse excitations and the receiving method to achieve high accuracy of sound source localization [8,9]. This mode makes the sonic source easy to distinguish from MA signals. However, the excitation is difficult to manufacture and poses safety risks. Also, it is susceptible to high-frequency pulse interference during the detection process [10]. These factors limit pulse mode detection accuracy, and the signal-to-noise ratio (SNR) is low.

Using the continuous wave mode in biological current detection [11] and electrical parameter measurements [12] achieves high detection accuracy [13], but it has not yet been used to locate the sonic source of currents or accurately reconstruct the neural current of biological tissue [7].

In this paper, the vector method is proposed to compute and analyze the MA signal using continuous modes under sinusoidal wave excitation. The paper poses a theoretical model and presents simulations of different models. The vector method is used to analyze the MA signal amplitude and phase characteristics of the sonic source. Experimental studies of wire sample validate the method.

This study sets a foundation for using low-frequency sinusoidal wave excitation with high accuracy and SNR to study the distribution imaging of tissue's electrical parameters. This method also aids in locating the sonic source of a biological current, and in reconstructing neural activity.

\section{Theory}

The magneto-acoustic effect is modeled by the wave equation. For the excitation $S(j \omega)$, the wave equation in the frequency domain is [14]

$$
\nabla^{2} \boldsymbol{P}(\boldsymbol{r}, j \omega)+\left(\omega^{2} / c^{2}\right) \boldsymbol{P}(\boldsymbol{r}, \omega)=(\nabla \cdot \boldsymbol{F}) S(j \omega) H(j \omega)
$$

Where $\boldsymbol{p}(\boldsymbol{r}, t)$ is the MA signal and $\boldsymbol{F}$ is the Lorentz force density in the sample. $\boldsymbol{F}=\boldsymbol{J} \times B_{0} . \boldsymbol{J}$ is the current density, $\boldsymbol{B}_{0}$ is the static magnetic field, and $c$ is the sample's sonic velocity. $H(j \omega)$ is the system function to the magneto-acoustic measuring system. [15]

The source term can be written as a product of a purely spatial and a purely temporal component

$$
\boldsymbol{P}(\boldsymbol{r}, j \omega)=-c S(j \omega) H(j \omega) \iiint_{V} d v(\nabla \cdot \boldsymbol{F}) \frac{e^{j \omega \mid \boldsymbol{r}-\mathbf{x}_{0} / c}}{4 \pi\left|\boldsymbol{r}_{0}-\boldsymbol{r}\right|}
$$

The vector method is based on the rotating vector method in circuit theory [16]. A sinusoidal signal $s(t)=A_{1} \sin \left(\omega_{1} t+\omega_{1} t_{1}\right)$ can be represented as a vector $A_{1}+j \omega_{1} t_{1}$ in a complex plane, as shown in Figure 1(a). The real part is $A_{1} \sin \left(\omega_{1} t_{1}\right)$; the imaginary part is $A_{1} \cos \left(\omega_{1} t_{1}\right)$.

When the sonic source moves, the distance varies from $L_{a}$ to $L_{b}$ and the vector rotates from $v_{a}$ to $v_{b}$. From Eq. (2) it can be deduced that the amplitude will decrease with $1 / L$, and phase $\Phi_{b^{-}} \Phi_{a}$ changes linearly because the transmitting time delay is linear to the distance.

$$
\Phi_{b}-\Phi_{a}=360^{\circ}\left(L_{b}-L_{a}\right) \omega / 2 \pi c
$$




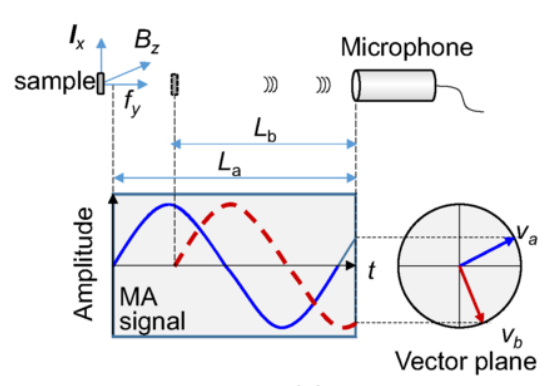

(a)

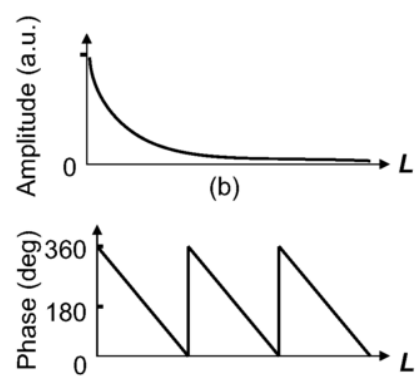

(c)

Fig. 1. Theory of magneto-acoustic signal measuring basing on the vector method.

If the medium is considered as a number of separate sonic sources, the summation vector corresponding to the MA signal formed by several sonic sources is the summation of the real and imaginary part for each vector in the complex plane. For the i-th discrete sonic source,

$$
\begin{gathered}
\boldsymbol{P}_{i}(r, j \omega)=\left(1 / L_{i}\right) \boldsymbol{f}(r, j \omega) A_{i} e^{j \omega l_{i} / c} \\
\boldsymbol{f}(r, t)=-(1 / 4 \pi) \pi c \nabla \cdot\left(\hat{\boldsymbol{J}} \times \boldsymbol{B}_{0}\right) \otimes h(t)
\end{gathered}
$$

The vector form is expressed as:

$$
\boldsymbol{P}_{i}(r, j \omega)=\operatorname{Re}_{P i}+j \operatorname{Im}_{P i}
$$

Where the real part and imaginary part are:

$$
\left\{\begin{array}{l}
\operatorname{Re}_{\boldsymbol{P}_{i}}=\left(1 / L_{i}\right) \boldsymbol{f}(r, j \omega) A_{i} \cos \left(j \omega_{1} L_{i} / c\right) \\
\operatorname{Im}_{\boldsymbol{P}_{i}}=\left(1 / L_{i}\right) \boldsymbol{f}(r, j \omega) A_{i} \sin \left(j \omega_{1} L_{i} / c\right)
\end{array}\right.
$$

We can get:

$$
\boldsymbol{P}(r, j \omega)=\sum_{i} \boldsymbol{P}_{i}(r, j \omega)=\sum_{i} \operatorname{Re}_{P_{i}}+j \sum_{i} \operatorname{Im}_{P_{i}}
$$

Therefore, the measured amplitude and phase of the MA signal in the continuous mode are:

$$
\begin{aligned}
A M P_{n} & =\sqrt{\left(\sum_{i} \operatorname{Re}_{P i}\right)^{2}+\left(\sum_{i} \operatorname{Im}_{P i}\right)^{2}} \\
P H A_{n} & =\arctan \left(\sum_{i} \operatorname{Im}_{P i} / \sum_{i} \operatorname{Re}_{P i}\right)
\end{aligned}
$$

\section{Method}




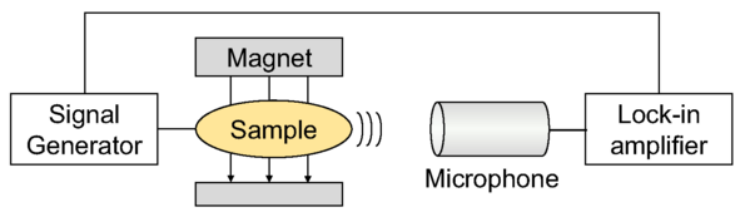

Fig. 2. Experimental setup.

\subsection{Simulating method}

A simulation to line model was used to verify the vector methods and analyze the MA signal characteristics. Based on the discussed above, the amplitude and phase can be calculated using Eqs. (9) and (10). The finite element method (FEM) of a Comsol Multiphysics platform was used in the numerical solution.

\subsection{Experimental method}

Figure 2 shows the experimental system. A signal generation was used to generate the sinusoid wave (AFG 3252, Tek Co., LTD). The sample was placed in a magnet's magnetic field. The flux density was 0.35 Tesla. A microphone (MP201, BSWA Co., LTD) received the MA signal. A lock-in amplifier (LI5640, NF Co., LTD) measured the amplitude and phase of the MA signal.

To validate the vector method and the simulation results, a copper wire sample was used in the preliminary experiments.

\section{Results}

\subsection{Simulating results}

\subsubsection{Single sonic source simulation}

To simulate the tissue surface or the electrical current, a linear sonic source was used as the simulating model. For a directional microphone, the linear source was simplified as a single source segment in the simulation.

For a single sonic source, the amplitude and phase of the MA signal from the sonic source with different positions, according to Eqs. (2) and (3), it is easily to deduce that, the amplitude decay with distance with $1 / L$ trend, while the phase varies linear with distance. The phase variation trend was different at different frequencies. For a simple single sonic source, simulation results and experimental results were compared in section 4.2.

\subsubsection{Two sonic sources simulation}

Two parallel current clusters were excited to simulate two tissue layers. When the two-layered boundary acoustic sources are $a$ and $b$, the amplitudes of are both $1 \mathrm{kgS}^{-2} \mathrm{~m}^{-3}$. A microphone was placed perpendicularly $1 \mathrm{~mm}$ away from sonic source $b$, and the distance between the microphone and the sonic source $a$ gradually increased from $1 \mathrm{~mm}$ to $100 \mathrm{~mm}$ at $1 \mathrm{~mm}$ steps. Figure 3 shows the simulation of the MA signal amplitude and phase at different distances.

The simulation results showed that vector $v_{b}$ corresponding to the MA signal from the sonic source $b$ exhibited a $1 / L$ decreasing amplitude trend as distance increasing; the phase decreased linearly with distance. Vector $v_{a}$ of sonic source $a$ was constant due to the amplitude and because the measuring 

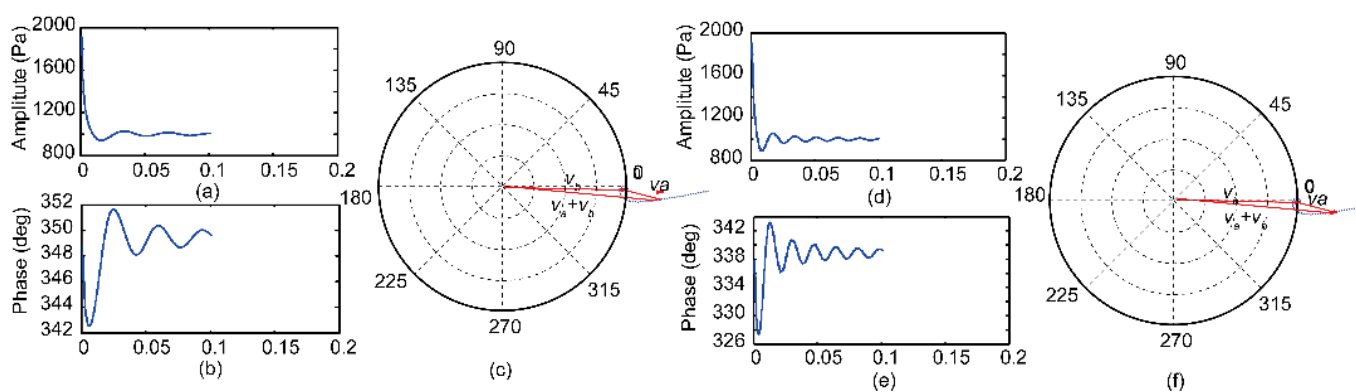

Fig. 3. Simulation results of two sonic sources.

Note: (a) is the simulated amplitude of the MA signal with different detecting distances under $10 \mathrm{kHz}$ excitation.
(b) is the simulated phase of the MA signal with different detecting distances under $10 \mathrm{kHz}$ excitation.
(c) is the vector analysis of (a) and (b) using the vector method.
(d) is the simulated amplitude of the MA signal with different detecting distances under $20 \mathrm{kHz}$ excitation.
(e) is the simulated phase of the MA signal with different detecting distances under $20 \mathrm{kHz}$ excitation.
(f) is the vector analysis of (d) and (e) using the vector method.

distance was unchanged.

The amplitude and phase showed periodic increases and decreases due to the rotating vector of $v_{b}$. The period was the same as the wave length excitation. The maximum and minimum amplitude values occurred at the phase differences of $180^{\circ}+\mathrm{n} \times 360^{\circ}$ and $\mathrm{n} \times 360^{\circ}(\mathrm{n}=0,1,2 \ldots)$. The ultimate values of the amplitude and phase equaled the $v_{b}$ because the $v_{a}$ amplitude was nearly zero due to $a$ is at infinity far.

Also, it can be seen that the trend was different at $10 \mathrm{kHz}$ and $20 \mathrm{kHz}$ excitation frequencies. The possible cause for this was that the high frequency made the $v_{a}$ changing period shorter than the low frequency.

\subsection{Experimental results}

\subsubsection{Single wired sample}

A $10 \mathrm{~mm}$-long wire was used as an experimental sample. The current was set to $20 \mathrm{~mA}$. The stimulating frequencies were $10 \mathrm{kHz}$ and $20 \mathrm{kHz}$. The flux density of the static magnetic field was 0.35 Tesla. The commercial microphones contained nickel, which was susceptible to the magnetization detection. The measuring distance from the sound source was set to $60 \mathrm{~mm}-125 \mathrm{~mm}$ in the experiments to avoid the magnetization effect.

As Figure 4 shows, different source locations have different phases formed by the propagating time delay. The phase curve period corresponded with the MA signal frequency. The experimental results are in accordance with the simulation. The phase change related to the frequency, which is in

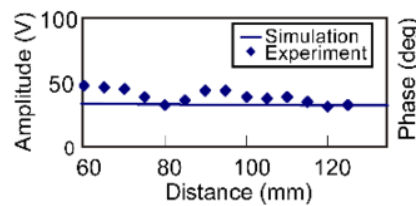

(a)

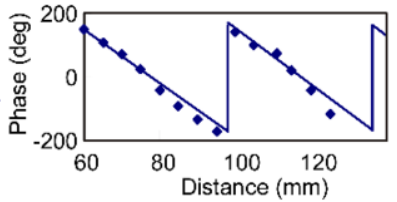

(b)

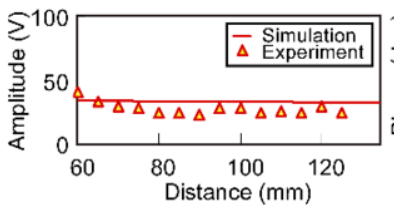

(c)

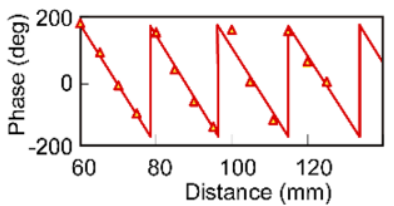

(d)

Fig. 4. Experimental result of the wire sample.

Note: (a) is the measured amplitude with different measuring distances. The excitation frequency was $10 \mathrm{kHz}$.

(b) is the measured phase with different measuring distances. The excitation frequency was $10 \mathrm{kHz}$.

(c) is the measured amplitude with different measuring distances. The excitation frequency was $20 \mathrm{kHz}$.

(d) is the measured phase with different measuring distances. The excitation frequency was $20 \mathrm{kHz}$. 
accordance with Eq. (3). The phase changes were $10.6^{\circ}$ and $21.2^{\circ}$ at $10 \mathrm{kHz}$ and $20 \mathrm{kHz}$, respectively, when the distance change was $1 \mathrm{~mm}$. The error between the theory and measured phases were $5.7 \%$ and $2.5 \%$ at two frequencies. The repeating periods were $37 \mathrm{~mm}$ and $17.2 \mathrm{~mm}$ of $10 \mathrm{kHz}$ and $20 \mathrm{kHz}$, respectively, which related to the frequency.

On the other hand, with increasing distance, the amplitude showed a $1 / L$ decay trend at both frequencies. The noise interference and attenuation created deviations between the experiments and the simulated value. This error can be reduced by shielding noise.

The results indicated that the sonic source location can be obtained by measuring the amplitude and phase of the MA signal.

\subsubsection{Multi-wired sample}

The MA signal of a set of three parallel wires was measured to study and validate the summing characteristic of the sonic sources.

A $10 \mathrm{~mA}$ current was applied to three copper wire samples. The exciting frequencies were $10 \mathrm{kHz}$ and $20 \mathrm{kHz}$. The intervals of No. 1, No. 2, and No. 3 sonic source were $5 \mathrm{~mm}$. Each wire was $10 \mathrm{~mm}$ long. The microphone pointed perpendicularly to the wire. The measuring distance of the three sonic sources were $100 \mathrm{~m}, 105 \mathrm{~mm}$, and $110 \mathrm{~mm}$.

Because the shape differences between the wired samples resulted in amplitude and phase errors, a calibration was taken to eliminate the difference by measuring the three wires at the same position.

Figure 5 shows the experimental results and theoretical calculations.

The experiment validated the rules of vector triangle, and the simulation results. The error was within $\pm 10 \%$ due to system noise and measurement errors.

Meanwhile, considering summed sources formed by $1+2$ and $2+3$, it can be seen that the summed amplitudes of $A_{1+2}$ and $A_{2+3}$, also the phases $\Phi_{1+2}$ and $\Phi_{2+3}$, can be seemed as an integral source moving $5 \mathrm{~mm}$. It means the amplitude and phase can be seemed as an integral source due to the same interval of the single source.

Also, the measured amplitude and phase at $10 \mathrm{kHz}$ and $20 \mathrm{kHz}$ were different, which implied that the sonic source could be reconstructed using multi-frequency measurements.

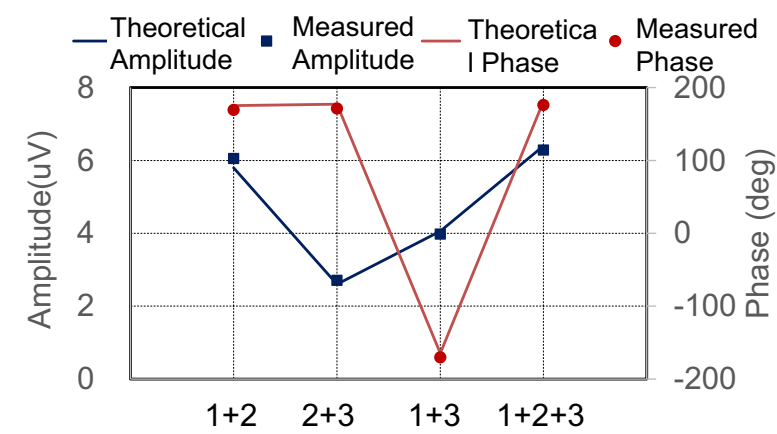

(a)

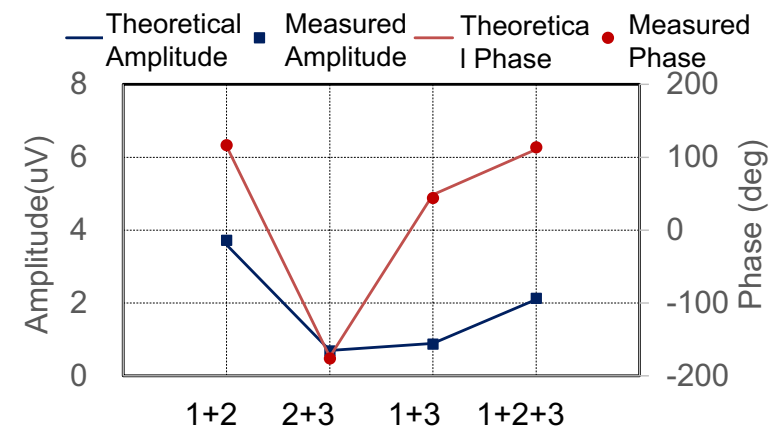

(b)

Fig. 5. Experimental results of the mi-sonic source.

Note: (a) is the measured amplitude and phase at $10 \mathrm{kHz}$.

(b) is the measured amplitude and phase at $20 \mathrm{kHz}$

The measured amplitudes were $3.60,2.61$, and 0.71 of sources a, b, and c at $10 \mathrm{kHz}$;

The measured phases were $179.98^{\circ}, 155.17^{\circ}$, and $-115.02^{\circ}$ of sources a, b, and c at $10 \mathrm{kHz}$;

The measured amplitudes were $2.01,2.20$, and 1.60 of sources a, b, and c at $20 \mathrm{kHz}$;

The measured phases were $95.10^{\circ}, 138^{\circ}$, and $-60.02^{\circ}$ of sources a, b, and c at $20 \mathrm{kHz}$. 


\section{Conclusion and discussion}

This paper presents a new vector method to determine MA signals. The paper also established a theoretical model and presents simulations. Experiments were conducted with copper wire samples to verify the theory and simulation.

The results showed that when using low-frequency continuous sinusoidal wave modes, the amplitude and phase of the MA signal contained sonic source distribution information. By measuring the amplitude and phase, the intensity and position of the sonic source can be measured and located. The results also verified the vector method and theory.

Follow-up work should be done in further studies. Firstly, real biological tissue experiments must be conducted to study the characteristics of the MA signal from the complex sonic source. Secondly, noise shielding should be improved to reduce measurement errors. Thirdly, based on the characteristics of the MA signal, research should be conducted on using the magneto-acoustic method to reconstruct the sonic source using amplitude and phase.

This study sets a foundation for studying the distribution imaging of tissue electrical parameters using low-frequency sine wave excitation with high accuracy and SNR. It also helps in locating the sonic source of a biological current through the magneto-acoustic method.

\section{Acknowledgment}

This work was supported in part by the Key Project of the National Natural Foundation of China (\#51137004, \#81127003), NNSFC (\#81171424), Tianjin Youth Fund in Basic Research on Application (\#13JCQNJC14000).

\section{References}

[1] H. Wen, J. Shah and R.S. Balaban, Hall effect imaging, IEEE Transactions on Biomedical Engineering 45 (1998), 119124.

[2] B. Roth and P. Basser, A theoretical model for magneto-acoustic imaging of bioelectric currents, IEEE Transactions on Biomedical Engineering 41 (1994), 723-728.

[3] J.F. Emerson, D.B. Chang, S. McNaughton, J.S. Jeong, K.K. Shung and S.A. Cerwin, Electromagnetic acoustic imaging, IEEE Transactions on Ultrasonics, Ferroelectrics and Frequency Control 60 (2013), 364-372.

[4] X. Sun, F. Zhang, Q. Ma, J. Tu and D. Zhang, Acoustic dipole radiation based, conductivity image reconstruction for magnetoacoustic tomography with magnetic induction, Applied Physics Letters 100 (2012), 024105.

[5] N. Narayana, Elements of Engineering Electromagnetics, China Machine Press, Beijing, China, 2006, pp. 35-37.

[6] M.S. Aliroteh, G. Scottt and A. Arbabian, Frequency-modulated magneto-acoustic detection and imaging, Electronics letters 50 (2014), 790-792.

[7] B. Roth, The role of magnetic forces in biology and medicine, Experimental Biology and Medicine 236 (2011), $132-137$.

[8] G. Hu, E. Cressman and B. He, Magnetoacoustic imaging of human liver tumor with magnetic induction, Applied Physics Letters 98 (2011), 023703.

[9] L. Mariappan, G. Hu and B. He, Magnetoacoustic tomography with magnetic induction for high-resolution bioimepedance imaging through vector source reconstruction under the static field of MRI magnet, Medical Physics 41 (2014), 022902.

[10] S. Wang, S. Zhang, R. Ma, T. Yin and Z. Liu, A study of acoustic source generation mechanism of magnetoacoustic tomography, Computerized Medical Imaging and Graphics 38 (2014), 42-48.

[11] H. Ammari, Y. Capdeboscq, H. Kang and A. Kozhemyak, Mathematical models and reconstruction methods in magneto-acoustic imaging, European Journal of Applied Mathematics 20 (2009), 303-17.

[12] M. Salim, E. Supriyanto, J. Haueisen, I. Ariffin, A.H. Ahmad and B. Rosidi, Measurement of bioelectric and acoustic 
profile of breast tissue using hybrid magnetoacoustic method for cancer detection, Medical \& Biological Engineering \& Computing 51 (2013), 459-466.

[13] B. Towe and M.R. Islam, A magneto-acoustic method for the noninvasive measurement of bioelectric currents, IEEE Transactions on Biomedical Engineering 35 (1988), 892-894.

[14] G. Hu and B. He, Magnetoacoustic imaging of electrical conductivity of biological tissues at a spatial resolution better than $2 \mathrm{~mm}$, Plos One 6 (2011), e23421.

[15] Y. Xu and B. He, Magnetoacoustic tomography with magnetic induction (MAT-MI), Institute of Physics Publishing 50 (2005), 5175-5187.

[16] E.C. Craig, Electronics via Waveform Analysis, Springer Verlag, US, 1993, pp. 315-317. 